This item was submitted to Loughborough's Research Repository by the author.

Items in Figshare are protected by copyright, with all rights reserved, unless otherwise indicated.

\title{
An incremental learning approach for physical human-robot collaboration
}

\section{PLEASE CITE THE PUBLISHED VERSION}

https://www.ukras.org/search/An+Incremental+Learning+Approach+for+Physical+Human-

Robot+Collaboration

PUBLISHER

EPSRC UK-RAS Network

VERSION

VoR (Version of Record)

LICENCE

CC BY-NC-ND 4.0

REPOSITORY RECORD

Buerkle, Achim, Ali Al-Yacoub, and Pedro Ferreira. 2020. "An Incremental Learning Approach for Physical Human-robot Collaboration”. Loughborough University. https://hdl.handle.net/2134/14135612.v1. 


\section{An Incremental Learning Approach for Physical Human-Robot Collaboration}

\author{
Achim Buerkle \\ Wolfson School of Engineering \\ Loughborough University \\ Loughborough, UK \\ a.buerkle@lboro.ac.uk
}

\author{
Ali Al-Yacoub \\ Wolfson School of Engineering \\ Loughborough University \\ Loughborough, UK \\ a.al-yacoub@lboro.ac.uk
}

\author{
Pedro Ferreira \\ Wolfson School of Engineering \\ Loughborough University \\ Loughborough, UK \\ p.ferreira@lboro.ac.uk
}

\begin{abstract}
Physical Human-Robot Collaboration requires humans and robots to perform joint tasks in a shared workspace. Since robot's characteristic strengths are to cope well with high payloads, they are utilized to assist human operators during heavy pulling or pushing activities. A widely used sensor to detect human muscle fatigue and thus, to trigger an assistance request, is an Electromyography (EMG). Many previous approaches to process EMG data are based on training Machine Learning models offline or include a large degree of manual fine tuning. However, due to recent advances in Machine Learning such as incremental learning, there is an opportunity to apply online learning which reduces programming effort and also copes well with subject specific characteristics of EMG signals. Initial results show promising potential, yet, unveil a conflict between convergence time and classification accuracy.
\end{abstract}

Keywords-EMG, Human-Robot Collaboration, Incremental Learning, Machine Learning

\section{INTRODUCTION}

Human-Robot Collaboration (HRC) in manufacturing aims to establish symbiotic or synergetic effects between human operators and robots [1]. This is enabled by combining the characteristic strengths of each party. Human strengths are considered to be adaptability to changes, decision making, and problem solving [1], [2]. Robot's strengths, on the other hand, are high precision, high operating speeds, and the capability of coping with high payloads [3]. Thus, in a physical collaboration, robots are able to support human operators via force amplification to handle heavy pushing and pulling activities [4]. In order to measure human muscle activity such as during the lift of heavy objects, a widely used sensor is an Electromyography (EMG) [5]. The approaches typically include pre-processing of the data, feature extraction, and a supervised leaning of the model [5], [6]. However, recent advances in Machine Learning regarding incremental learning could allow to minimize the training and programming effort of such models [7]. Furthermore, the algorithm could optimize its performance over time in an online system [7]. In this work, an incremental learning approach is utilized to predict EMG data during three different states: Participants lifting light payloads, medium payloads, and heavy payloads (struggling).

\section{RELATED WORK}

In a Human-Robot collaborative scenario, humans and robots perform joint tasks in a shared workspace [1]. In order to communicate intentions of the human to the robot, sensors are utilized such as EMGs [5]. The EMG signals are usually acquired from a human upper-limb since they are mostly used in the given tasks [5]. The acquired data can be used to communicate movement intentions. It can also provide insights on human muscle fatigue [6]. In this case, a robot

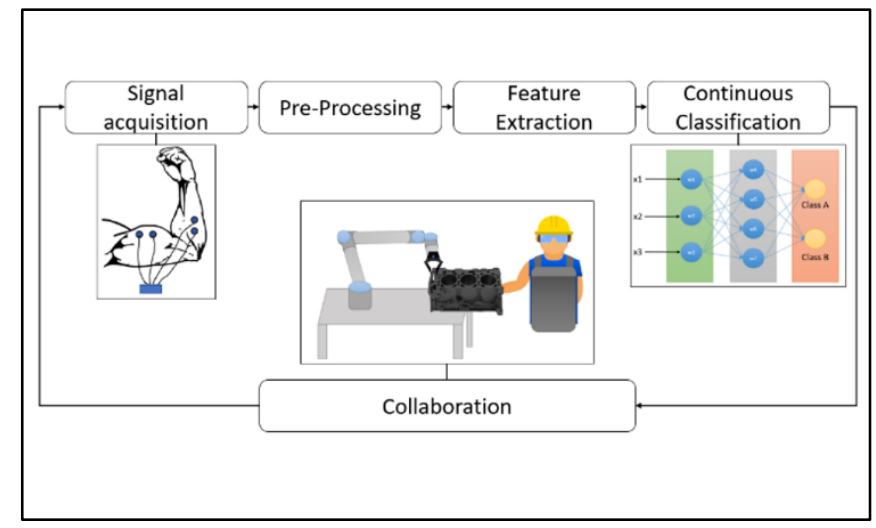

Figure 1 EMG Signal Processing for HRC (adapted) [5]

could assist a human operator during a heavy pull or push of an object or adapt its behavior to create more ergonomic working conditions for its human co-worker [6], [8]. This is intended to prevent injuries, as well as long-term health issues related to physical fatigue [8].

Figure 1 shows the general process used to integrate EMGs in Human-Robot Collaboration for a supervised, nonincremental learning approach. The first stage is EMG data acquisition. Critical attention is required during the selection of the acquisition device, the number of channels used, as well as the placement of each channel [5]. The channel acquisition device also determines the sampling rate and data transmission [9].

During the pre-processing stage, raw EMG signals are checked for baseline offset [5]. Typically, the signal is corrected by subtracting the average amplitude from each instance, however, there are also approaches based on nonlinear error-modelling [5], [6]. Raw EMG signals are susceptible to contain noise. Thus, Butterworth filters with a cut off frequency from $2 \mathrm{~Hz}-20 \mathrm{~Hz}$ are utilized [5]. The remaining features are extracted in the Feature selection and extraction stage. This is critical during EMG data processing since it has a high impact on the classification accuracy [9]. Three properties are considered as essential: class separability (minimize overlap), robustness (separability in noisy environment), and computational complexity (low complexity of features implies lower processing times) [5], [9].

The fourth stage is continuous classification of the filtered and extracted signals. There are mainly two types of prediction models. One is the use of kinematic models, the second approach is to utilize Artificial Neural Networks (ANNs) [5]. However, [9] states that Linear Discriminant Analyses (LDA) and Support Vector Machines (SVMs) are also widely used for EMG data classification. According to [5] there are few critical challenges remaining. Firstly, many 
offline systems obtain high classification accuracies, yet the online performances of such systems are far from satisfactory. Secondly, there are subject-specific characteristics of EMG signals. This can even include variation of the EMG signals for the same person during different recording sessions. An opportunity to increase the performance and to lower programming and fine-tuning effort could be incremental learning. Incremental learning algorithms have the following characteristics: ability of life-long learning, ability to incrementally tune the model's performance, and no prior knowledge about the data and its properties is needed [7].

\section{EXPERIMENTAL SETUP}

The experimental setup aims to collect EMG data during three different stages: light payload, medium payload, and high payload, during which a participant is slightly struggling.

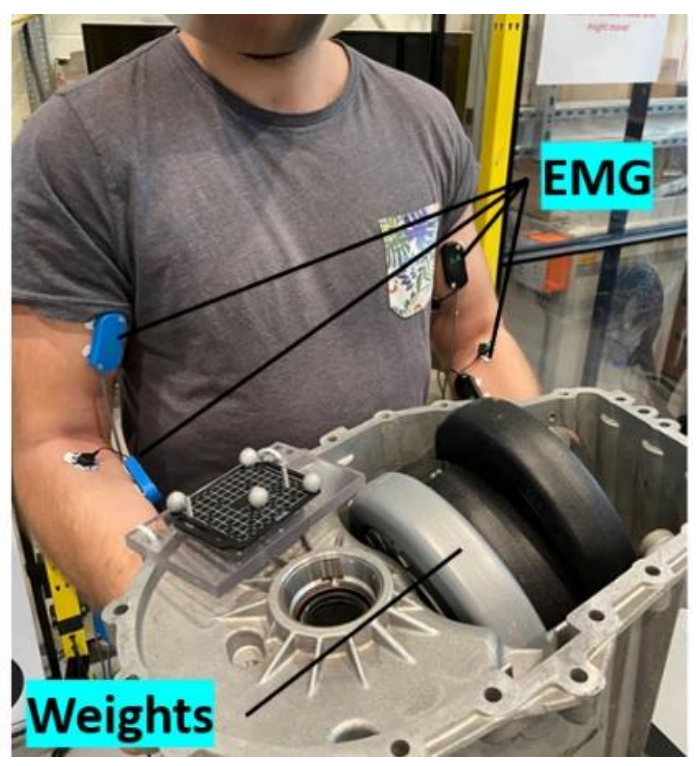

Figure 2 Experimental Setup

The acquired data will be fed into the classifier unlabeled. However, in order to validate the prediction results, predicted classes and the actual classification will be compared.

\section{RESUlTS AND DISCUSSION}

The collected data was used to train an Online Random Forest (ORF) model, that aims to classify the EMG signals into low payload, medium payload, and heavy payload. In any incremental learning approach, the most crucial property apart

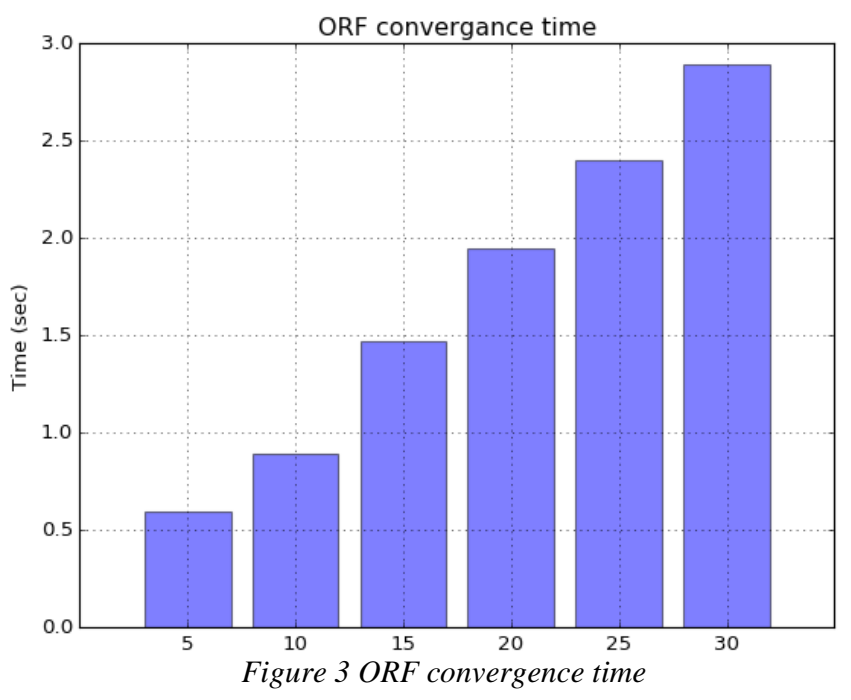

from accuracy is the convergence time. Since the model aims to minimize the prediction error live and immediately. In Human-Robot Collaboration, this is exceptionally important as humans and robots are physically interacting. Hence, in this validation experiment convergence time of the ORF model was measured with a different number of trees, which is illustrated in Figure 3.

As, expected, Figure 3 shows that the convergence time is directly proportional to the number of trees in the ORF model. The correspondent accuracy of the models in Figure 3 is shown in Table 1. The collected data for this experiment is $\sim 6000$ data points of EMG signals and the associated labels. Based on Figure 3 and Table 1, it can be noticed that the model must achieve a trade-off between accuracy and convergence time. The ORF model with 20 trees seems to be the most suitable model since it can converge in less than 2 seconds, and it achieves the highest detection accuracy.

Table 1 Number of Trees vs Prediction Accuracy

\begin{tabular}{|c|c|c|c|c|c|c|}
\hline $\begin{array}{c}\text { Number of } \\
\text { Trees }\end{array}$ & 5 & 10 & 15 & 20 & 25 & 30 \\
\hline $\begin{array}{c}\text { Mean } \\
\text { Square Error }\end{array}$ & 0.26 & 0.19 & 0.250 & 0.14 & 0.15 & 0.17 \\
\hline $\begin{array}{c}\text { Accuracy } \\
{[\%]}\end{array}$ & 82.3 & 84.6 & 85.2 & 89.7 & 86.7 & 86.7 \\
\hline
\end{tabular}

\section{CONCLUSION AND FUTURE WORK}

A novel incremental learning approach was introduced to determine physical workload from EMG data in HumanRobot Collaboration. During the online training, a conflict became clear between processing speed and accuracy. Lesser trees in the model meant faster convergence, however, it also resulted in the aforementioned lower accuracy. Overall, the accuracy could reach $89 \%$ in only two seconds. Thus, in a Human-Robot Collaborative Scenario this would allow the system to recognize a human operator struggling with the payload. The collaborative robot could then support the operator and subsequently, create a more ergonomic environment. However, prior to this technology being ready to be used in a practical application, further testing is essential. This includes the need for a larger sample size in participants and a richer variety in lifting tasks. The current setup allows to detect muscle contraction in participant's forearms and biceps. Yet, more EMG sensors placed on other muscle groups such as triceps and shoulders are expected to provide better results for predicting pushing activities. Furthermore, the system could be trained to not only detect temporary high payloads but also to recognize muscular fatigue during endurance tasks. This could help to improve human operator's posture and subsequently prevent negative long-term health effects.

Nevertheless, early results of this incremental learning approach demonstrate a reduced manual fine-tuning effort and it coping well with subject specific characteristics in the data. This offers the potential to be applied for additional human sensor technologies and subsequent data classifications. Ultimately, this could help to make Human-Robot Collaboration safer and more efficient. 


\section{REFERENCES}

[1] L. Wang et al., "Symbiotic human-robot collaborative assembly," CIRP Ann., 2019.

[2] V. Villani, F. Pini, F. Leali, and C. Secchi, "Survey on human-robot collaboration in industrial settings: Safety, intuitive interfaces and applications," Mechatronics, no. June 2017, pp. 1-19, 2018.

[3] J. Krüger, T. K. Lien, and A. Verl, "Cooperation of human and machines in assembly lines," CIRP Ann. - Manuf. Technol., vol. 58, no. 2, pp. 628-646, 2009.

[4] J. Schmidtler and K. Bengler, "Fast or Accurate? Performance Measurements for Physical Humanrobot Collaborations," Procedia Manuf., vol. 3, no. Ahfe, pp. 1387-1394, 2015.

[5] L. Bi, A. Feleke, and C. Guan, "A review on EMGbased motor intention prediction of continuous human upper limb motion for human-robot collaboration," Biomed. Signal Process. Control, vol. 51, pp. 113-127, 2019.

[6] L. Peternel, C. Fang, N. Tsagarakis, and A. Ajoudani, "A selective muscle fatigue management approach to ergonomic human-robot co-manipulation," Robot. Comput. Integr. Manuf., vol. 58, no. January, pp. 6979, 2019.

[7] A. Bouchachia, B. Gabrys, and Z. Sahel, "Overview of some incremental learning algorithms," IEEE Int. Conf. Fuzzy Syst., no. August, 2007.

[8] L. Peternel, N. Tsagarakis, D. Caldwell, and A. Ajoudani, "Robot adaptation to human physical fatigue in human-robot co-manipulation," Auton. Robots, vol. 42, no. 5, pp. 1011-1021, 2018.

[9] M. Hakonen, H. Piitulainen, and A. Visala, "Current state of digital signal processing in myoelectric interfaces and related applications," Biomed. Signal Process. Control, vol. 18, pp. 334-359, 2015. 